\title{
METODOLOGÍA INTEGRAL DE DIAGNÓSTICO DE ESTRUCTURAS DE CONCRETO REFORZADO
}

\author{
L. EBENSPERGER ${ }^{1}$, J. P. DONOSO ${ }^{2}$ \\ ${ }^{1}$ Doktor Ingenieur, Consultor en Tecnología del Concreto, Construtechnik Ltda., Chile \\ ${ }^{2}$ Ingeniero Civil, BDL Soluciones Estructurales de Ingeniería y Consultoría, Chile
}

\begin{abstract}
RESUMEN
Se presenta una metodología que mediante la utilización de ensayos no-destructivos ejecutados in-situ, entrega como resultado un análisis integral del estado de una estructura de concreto reforzado con información fundamental para la gestión de mantenimiento. La integración de los resultados en el Modelo CTK-ConDiag®, considerando una clasificación por severidad según ensayo y agresividad de la exposición solicitante, permite obtener un Índice Global de Deterioro de la Estructura. Considera la ejecución de ensayos de esclerometría, de ubicación, diámetros y espesores de recubrimiento de las barras de refuerzo existentes mediante técnicas GPR, del estado de ellas mediante la medición de la Velocidad y/o Potencial de Corrosión, y la calidad del recubrimiento de concreto a través del ensayo de Permeabilidad al Aire.
\end{abstract}

Palabras clave: Concreto, durabilidad, ensayos no-destructivos, Deterioro Estructural.

\begin{abstract}
A methodology is presented that through the use of non-destructive tests executed on-site conditions, results in an integral quantitative analysis of the state of a reinforced concrete structure. This allows for fundamental information to adjust maintenance cycles or make decisions on time in critical situations. It considers the execution of rebound hammer tests, determining the state of reinforcement by measuring the speed and/or corrosion potential, and the location, diameters and thicknesses of concrete cover using GPR techniques. The incorporation of the results in the CTK-ConDiag ${ }^{\circledR}$ Model allows obtaining a Global Index of Deterioration of the Structure, which considers a ranking of severity for each test and also for the aggressiveness of the acting exposure.
\end{abstract}

Keywords: Concrete, durability, nod-destructive testing NDT, Structural Damage.

\section{RESUMO}

A metodologia aplicada foi de testes não destrutivos e executados in-situ, que como resultado obtiveram uma análise quantitativa integral do estado de uma estrutura de concreto reforçado. Através de tais testes se conseguiu informações fundamentais que ajustaram os ciclos da manutenção ou para ter um período hábil para poder decidir o que fazer antes das situações críticas.

Considera o desempenho dos testes de Esclerômetria, o estado das armaduras de reforço através da medição da Velocidade e/ou Potencial da corrosão, e da localização, diâmetros e espessuras de revestimento aplicando técnicas de GPR. A incorporação dos resultados no Modelo CT-ConDiag® permite a obtenção de um Índice Global de Deterioração da Estrutura, que respeita o ranking da severidade por cada teste e por cada da intensidade da exposição actuante.

Palavras-chave: Concreto, durabilidade, testes não destrutivos, deterioração estrutural. 


\section{INTRODUCCIÓN}

El diagnóstico de obras de concreto reforzado, con la finalidad de determinar patologías habituales, las cuales pueden afectar las características de resistencia y serviciabilidad de una obra desde el punto de vista del usuario final, ha tomado en los últimos años una importancia relativa mayor, dado el aumento exponencial que han tenido a nivel mundial las reparaciones de estructuras de concreto y más aún el costo de estas, el cual se incrementa cada vez más.

El nivel de serviciabilidad se traduce en que por distintas causas que generan un deterioro, la Vida Útil de la estructura se ve afectada. Se entiende por Vida Útil el período de tiempo especificado en el diseño de la estructura, para el cual la estructura o parte de ella puede ser utilizada para el propósito previsto, no siendo necesario reparaciones mayores. Lo anterior queda reflejado en los siguientes ejemplos que se muestran en la Figura 1.

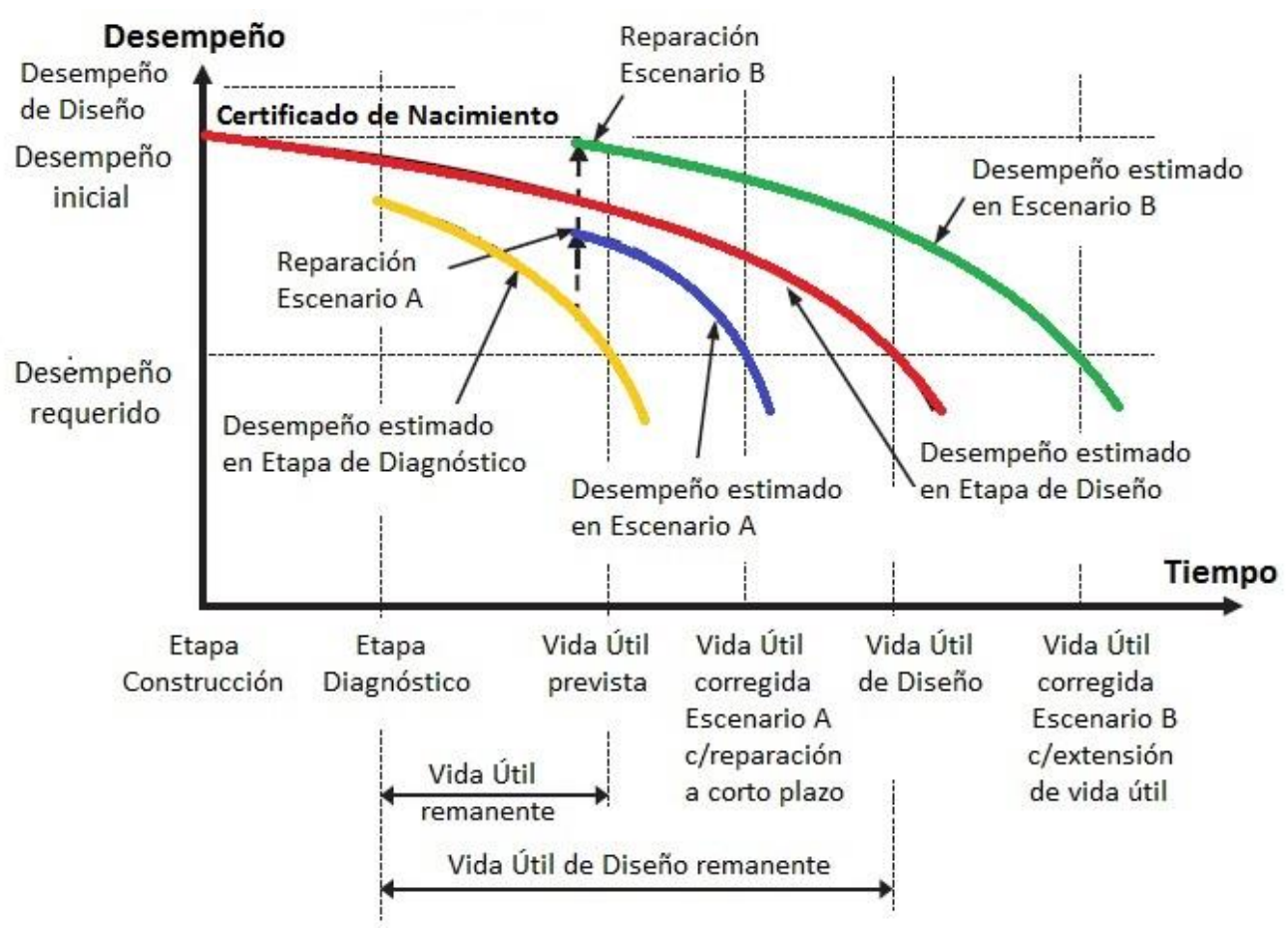

Figura 1: Nivel de Serviciabilidad (Desempeño) de una estructura en el tiempo. Adaptado de (ISO 16311, 2014).

Por una condición natural de envejecimiento, ocurre un deterioro paulatino con la edad, con la consecuente disminución del nivel de desempeño esperado o de Diseño de la obra (curva roja). La ejecución de un Diagnóstico luego de transcurrido un tiempo razonable, de acuerdo con el nivel de agresividad o de daños observados en una estructura, permitirá identificar, si el desempeño real de la estructura coincide o no con el de Diseño: la curva amarilla indica un desempeño menor.

De no ejecutar ninguna acción, la Vida Útil remanente se ve drásticamente reducida (cruce de curva roja y amarilla con Nivel de Desempeño requerido); es necesario ejecutar trabajos de mantención, los cuales pueden ser parciales o reducidos (Escenario A curva azul) o exhaustivos (Escenario B curva verde, con mayor costo). En el primer caso se aumenta la Vida Útil remanente, pero se mantiene por debajo de la de Diseño. En cambio, el Escenario B permite incluso extender la Vida Útil original del proyecto.

La condición de "Desempeño Inicial" puede ser determinada mediante la ejecución de ensayos a ejecutarse sobre la obra terminada, quedando incorporado en el "Certificado de Nacimiento" de la estructura. 
La ejecución de Diagnósticos para determinar la condición actual de estructuras existentes es esencial para respaldar el proceso de toma de decisiones con respecto a su conservación y mantención en el tiempo, y determinar además la Vida Residual o Remanente de ésta.

\section{EXPERIENCIAS Y NORMATIVAS INTERNACIONALES}

En la concepción del proyecto que se presenta se consideraron 3 Etapas de investigación:

1) Revisión Bibliográfica

- Consistente en una búsqueda acuciosa de información a nivel mundial acerca de normativas y experiencias con respecto al Diagnóstico, Mantenimiento y Modelación.

2) Desarrollo de Modelo de Diagnóstico Integral

- Considerando las experiencias recopiladas, se propone un Modelo que permite consolidar la información adquirida mediante distintos ensayos no-destructivos, buscando un Índice de Deterioro Global de una Estructura.

3) Desarrollo de Programa de Ejecución de Ensayos en 2 puentes

- Ejecutar en condiciones reales de terreno series de ensayos que permitan validar el Modelo presentado para el Análisis Integral de los Resultados. Se elegirán 2 puentes con distintas Exposición Ambiental (ambiente de cloruros cerca del mar, ambiente con efecto de $\mathrm{CO}_{2}$ ).

El avance actual del proyecto incluye las 2 primeras Etapas, habiéndose planificado la Etapa 3 para ser ejecutado durante los próximos 6 meses.

\subsection{Revisión Bibliográfica en Diagnóstico}

En el año 2014 se publica la norma internacional ISO 16331 sobre "Mantenimiento y Reparación de Estructuras de Concreto", entregando lineamientos estandarizados con principios generales y específicos que rigen la totalidad de las actividades relacionadas a estos temas. Estos lineamientos constan de 4 partes, según se indica a continuación:
o Parte 1:
Principios Generales
○ Parte 2:
Diagnóstico de estructuras de concreto existentes
○ Parte 3:
Diseño de reparaciones y prevención
- Parte 4:
Ejecución de reparaciones y prevención

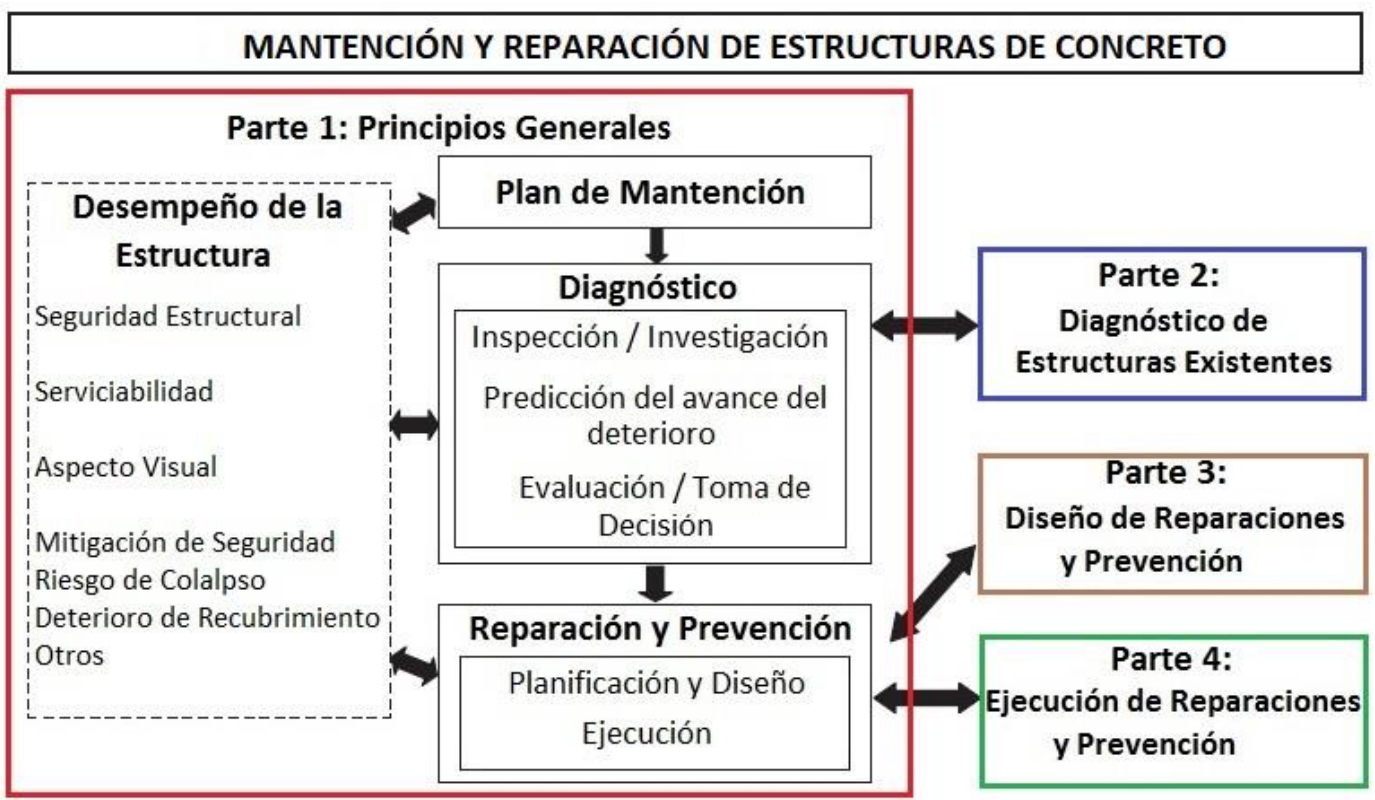

Figura 2: Flujograma de actividades relacionadas a la Mantención y Reparación de Estructuras 
Básicamente, se desprende de este documento que el Diagnóstico es una parte fundamental de todo proceso de Mantención y Reparación de estructuras. Como tal, deberá incorporar aspectos de Desempeño, Prevención y Diseño junto a la Ejecución de Reparaciones, entre otros, tal como lo muestra el Flujograma de la Figura 2.

Por otro lado, el American Concrete Institute ACI publica desde el año 2007 al 2013 tres Guías con Recomendaciones, las cuáles abarcan los procesos de Evaluación previo a la Rehabilitación (ACI 364.1 R-07, 2007), Ejecución de Inspección Visual (ACI 201.1 R-08, 2008) y Código de Requisitos para la Evaluación, Reparación y Rehabilitación de Edificaciones (ACI 562-13, 2013):

- El primero de ellos se presenta como una serie de recomendaciones y directrices, basadas en la experiencia extraída de fuentes e investigaciones pasadas. Las pautas dadas en este informe son de carácter general, pero lo suficientemente específicas como para modelar un Procedimiento de Evaluación de Estructuras de Concreto.

- A su vez la Guía de Inspección Visual se orienta específicamente a una de las primeras etapas de un Diagnóstico. La inspección visual es un examen de la estructura para identificar y definir las condiciones en que se encuentran las superficies de concreto que son visualmente accesibles. Al completar una inspección visual del concreto de inmediato después de la construcción, y mediante la repetición a intervalos durante la vida útil, proporciona información histórica importante sobre el desempeño y durabilidad. Los resultados de la inspección también ayudan a la detección temprana de peligro y deterioro, disminución de parámetros de riesgo, permitiendo la reparación a costo menor o la rehabilitación antes de que una demolición o reemplazo sea necesario.

- Por último, el Código está orientado solamente a Edificaciones y ciertas estructuras civiles de Hormigón, como arcos, estanques, depósitos, silos, estructuras resistentes a explosiones e impactos, chimeneas y fundaciones, como placas de fundaciones, pilotes de hormigón, cajones enterrados y muelles. Es complementario al Código ACI 318-14 sobre Diseño Estructural. Proporciona los requisitos mínimos para la" Evaluación, Reparación, Rehabilitación y Refuerzo de edificios de concreto", pero no proporciona procedimientos de diseño completos o prácticas constructivas. Busca interrelacionar las reparaciones con los requisitos ya establecidos en la estructura original. El código comprende requisitos prescriptivos y por desempeño, entregando un comentario para ambos conceptos. Está destinado para proporcionar orientación al profesional y está referenciado a las correspondientes fuentes de información.

La Asociación Americana de Carreteras preparó (AAHSTO, 2011) un extenso Manual para la Evaluación de Puentes. Éste constituye un documento completo de los puntos a seguir al momento de evaluar alguna estructura. Este manual ha sido desarrollado para ayudar a los propietarios de puentes, que por lo general el Estado, o en la actualidad también empresas concesionarias, estableciendo procedimientos de inspección y prácticas de evaluación que cumplen con los estándares de inspección de la NBIS 1 .

Esta organización cuenta además con su código LRDF de Diseño de Puentes (AAHSTO, 2017) para la Especificación del Diseño y Construcción de Puentes Carreteros, basado en aspectos de confiabilidad de la estructura. Se describen variados aspectos de diseño, como solicitaciones y factores de carga para construcciones con distintos materiales, entre otros, además de aspectos de evaluación y rehabilitación. En Japón, el Instituto Japonés del Concreto JCI (Imamoto, 2016) publicó una novedosa Guía para la Evaluación de Estructuras de Concreto existentes, la cual cubre los requisitos generales, métodos y procedimientos para la evaluación de estructuras, desde edificaciones hasta estructuras industriales y puentes, incluyendo estructuras armadas y pretensadas, y aquellas que han sido rehabilitadas. El objetivo principal de esta Guía es entregar directrices para la evaluación de la confiabilidad (nivel de confianza) de una estructura existente en relación con el desempeño requerido para su uso futuro.

\footnotetext{
${ }^{1}$ National Bridge Inspection Standards
} 
A través de la definición de 3 Niveles de Evaluación y 3 Grados de Precisión, asociados a una Condición o Criterio a evaluar que se encuentra clasificado a su vez en otros 3 Niveles, la metodología entrega un interesante mecanismo de establecimiento de las condiciones actuales de una estructura, con tal de poder confrontarlas con los criterios de verificación.

El Nivel I se centra en la revisión de documentación, especificaciones técnicas y una inspección visual básica. La predicción de las propiedades futuras las realiza a través de lo establecido en códigos de diseño. El Nivel II, en cambio, pasa a una inspección detallada con cantidad limitada de muestras y medición directa con ensayos no-destructivos y semi-destructivos, y la predicción de propiedades de los materiales con ensayos de desempeño a testigos, considerando revisiones estructurales posteriores utilizando códigos de diseño actuales y datos de propiedades de los materiales medidos anteriormente. En el Nivel III se procede a ejecutar análisis numéricos avanzados como FEM estableciendo criterios de verificación según el desempeño requerido, usando las propiedades reales actuales de los materiales, pudiendo estos resultados compararse a través del código vigente a la fecha de construcción, o bien, idealmente, con el código vigente actual de diseño.

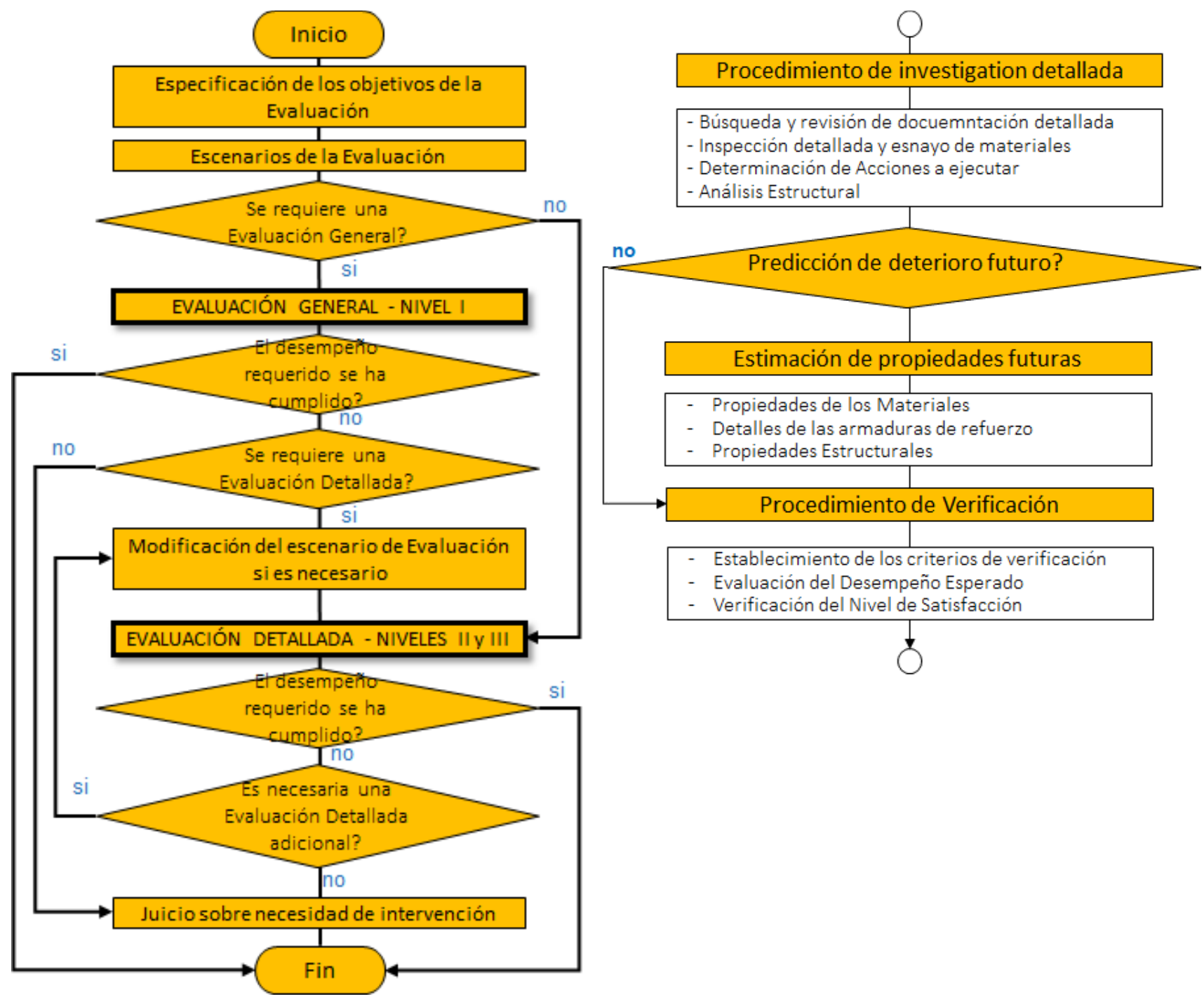

Figura 3: Flujograma General y Detallado de Evaluación de Estructuras existentes según JCI

El Diagnóstico comienza con una Investigación General (Nivel I), en la cual se realiza una evaluación del desempeño de la estructura en aspectos de Serviciabilidad, Durabilidad y Seguridad Estructural. Cada aspecto es identificado con una letra y una condición, que se clasifica en 3 Clases: 
Tabla 1: Condiciones y Clases utilizadas en la Evaluación de Desempeño según JCI

\begin{tabular}{|c|c|c|c|c|c|}
\hline \multicolumn{2}{|l|}{$\begin{array}{c}\text { Ítems de } \\
\text { Evaluación de } \\
\text { Desempeño }\end{array}$} & Condiciones & $\begin{array}{c}\text { Clase } 1 \\
\text { situación } \\
\text { menos } \\
\text { desfavorable }\end{array}$ & $\begin{array}{c}\text { Clase } 2 \\
\text { situación } \\
\text { intermedia }\end{array}$ & $\begin{array}{c}\text { Clase } 3 \\
\text { situación más } \\
\text { desfavorable }\end{array}$ \\
\hline \multirow{3}{*}{$\begin{array}{l}\text { Serviciabilidad / } \\
\text { Durabilidad }\end{array}$} & $\mathbf{V}$ & Vida Útil transcurrida & V1 & $\mathrm{V} 2$ & V3 \\
\hline & W & Vida Útil Residual & W1 & W2 & W3 \\
\hline & $\mathbf{X}$ & $\begin{array}{l}\text { Ambiente y Condiciones de } \\
\text { Servicio }\end{array}$ & $\mathrm{X} 1$ & $\mathrm{X} 2$ & $\mathrm{X} 3$ \\
\hline \multirow{2}{*}{$\begin{array}{l}\text { Seguridad } \\
\text { Estructural }\end{array}$} & $\mathbf{Y}$ & $\begin{array}{l}\text { Diseño / Ejecución / } \\
\text { Mantención }\end{array}$ & Y1 & $\mathrm{Y} 2$ & Y3 \\
\hline & $\overline{\mathbf{Z}}$ & Deterioro / Daño & $\mathrm{Z1}$ & $\mathrm{Z2}$ & $\mathrm{Z3}$ \\
\hline
\end{tabular}

La combinación entre condiciones y Clases para cada estructura en evaluación entregará finalmente como resultado que:

- La condición actual es aceptable

- Existe una necesidad de seguimiento

- Se recomienda una Evaluación detallada (Nivel II o III)

- Se requiere una acción inmediata

Desde el año 2015 y por 4 años sesionó un grupo de expertos europeos interdisciplinario, asociados bajo un Acción de la Comunidad Europea (COST Action TU1406, 2018) con el objetivo de estandarizar a nivel europeo las especificaciones para el Control de Calidad de estructuras de puentes. Las recomendaciones y directrices definidas no son prescriptivas, pero recomiendan y permiten una amplia gama de metodologías ya en aplicación en varias redes de puentes. El proceso busca establecerse evaluando indicadores de desempeño apropiados y mejorando la planificación de estrategias de mantenimiento (ver Figura 4):

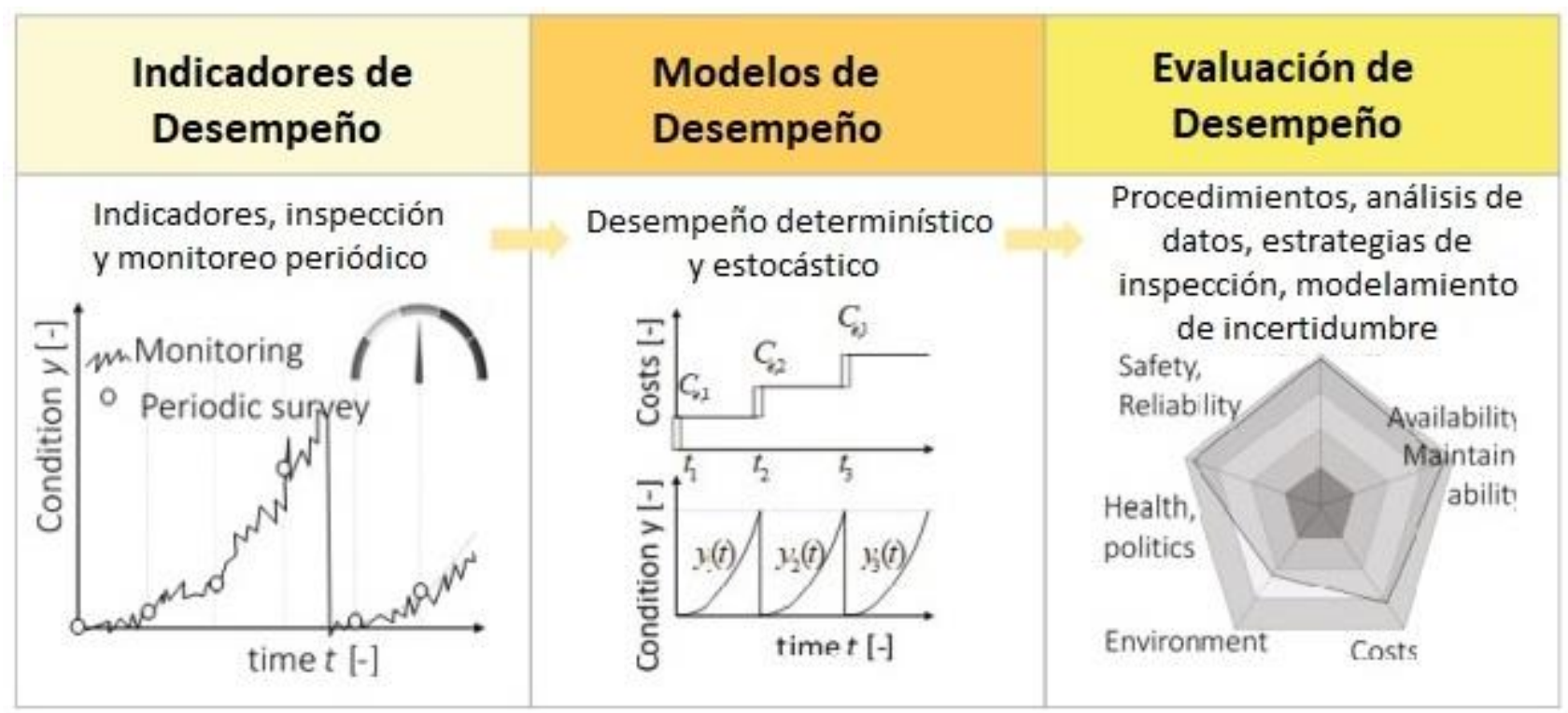

Figura 4: Idea conceptual de la gestión de puentes de carretera a través de a) Indicadores de Desempeño, que conducen a b) Modelos de Desempeño, que posteriormente se asignan a c) Evaluación de Desempeño basada en múltiples criterios (COST Action TU1406, 2018).

En este contexto, un primer paso ha sido el establecimiento de recomendaciones específicas para la evaluación de puentes de carreteras en forma de identificación de los métodos utilizados para la 
cuantificación de indicadores de desempeño. Un segundo paso es la definición de objetivos de desempeño estandarizados. Finalmente, el desarrollo de una guía para el establecimiento de planes de Calidad para puentes de carreteras. Los modelos de predicción se enfatizan aquí como un factor de influencia clave. Además, se destaca el concepto de gestión sustentable mediante la evaluación de indicadores de desempeño ambiental, económico y social durante todo el ciclo de vida.

Los Indicadores de Desempeño PI abordan los siguientes objetivos:

- Seguridad Estructural: factor de carga, el índice de confiabilidad en Estado Límite Último;

- Serviciabilidad: índice de condición, índice de confiabilidad para Estado Límite de Servicio;

- Disponibilidad, robustez;

- Costos: el costo total del Ciclo de Vida de la Estructura, valores relacionados con aspectos de durabilidad;

- Eficiencia ambiental: huella de CO2.

Los PI capturan las propiedades mecánicas y técnicas y su comportamiento de degradación, que son actualmente, en parte, cubiertos por las especificaciones de los códigos. Los aspectos considerados incluyen el envejecimiento natural; la calidad del material; métodos de diseño de vida útil; índices de sustentabilidad; índices ambientales, económicos y sociales y perfiles de desempeño. La Figura 5 presenta los Tipos de Indicadores de Desempeño presentes considerados en estas Recomendaciones.

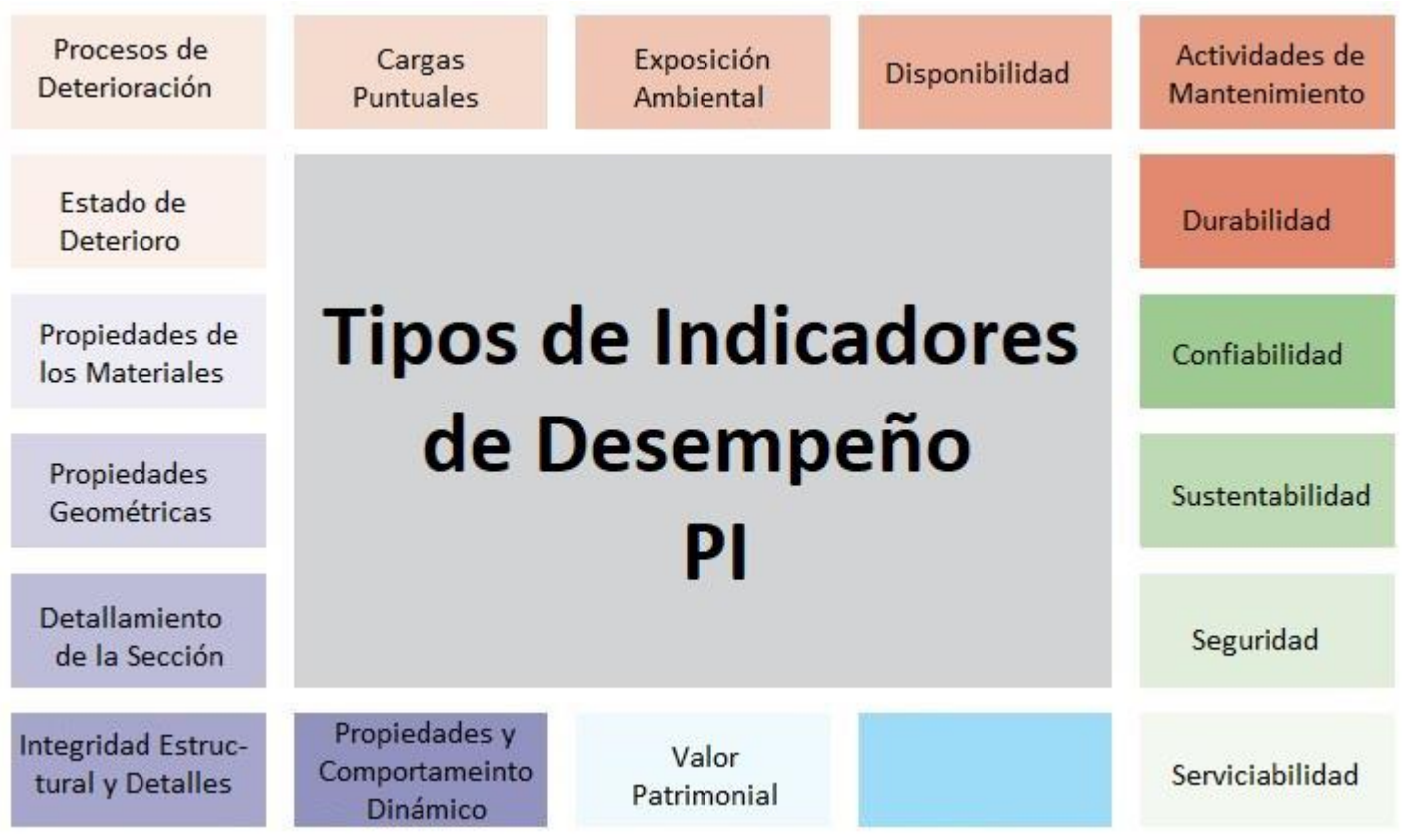

Figura 5: Tipo de Indicadores de Desempeño considerados en la Metodología (COST Action TU1406, 2018).

La aplicación de los PI se realiza en 3 niveles:

- Componentes (sub-, superestructura)

- Sistema (importancia de los elementos y funcionalidad del Puente), y

- Red Global de Carreteras (importancia del puente y funcionalidad de la Red)

Es establecimiento de un Plan de Control de Calidad y los respectivos Objetivos de Desempeño definen las consecuencias y acciones requeridas en cada unidad en estudio.

El análisis multi-criterio propuesto en la Metodología lleva a contar con Indicadores de Desempeño Claves KPI, los cuales son graficados en un diagrama tipo "araña" (ver Figura 6), donde la escala 1 representa la situación más favorable, y la escala 5 la más desfavorable. La clasificación definida es la siguiente: 
- Confiabilidad: la probabilidad de que el puente sea adecuado para su propósito durante su vida útil. Es el complemento a la probabilidad de la falla estructural (es decir, seguridad), falla operacional (es decir, serviciabilidad) o cualquier otro modo de falla.

- Disponibilidad: la proporción de tiempo que un sistema está en condiciones de funcionamiento. Se origina a partir de intervenciones de mantenimiento planificadas (por ejemplo, tiempo de viaje adicional debido a un régimen de tráfico impuesto en el puente).

- Seguridad: relacionada con minimizar o eliminar el daño a las personas durante la vida útil de un puente. La pérdida de vidas debidas al fallo estructural no está incluida.

- Economía: relacionada con minimizar los costos a largo plazo y las actividades de mantenimiento durante la vida útil de un puente. Aquí el costo del usuario incurridos debido a desvíos y retrasos no están incluidos.

- Medio Ambiente: asociado con minimizar el daño al medio ambiente durante la vida útil de un puente.

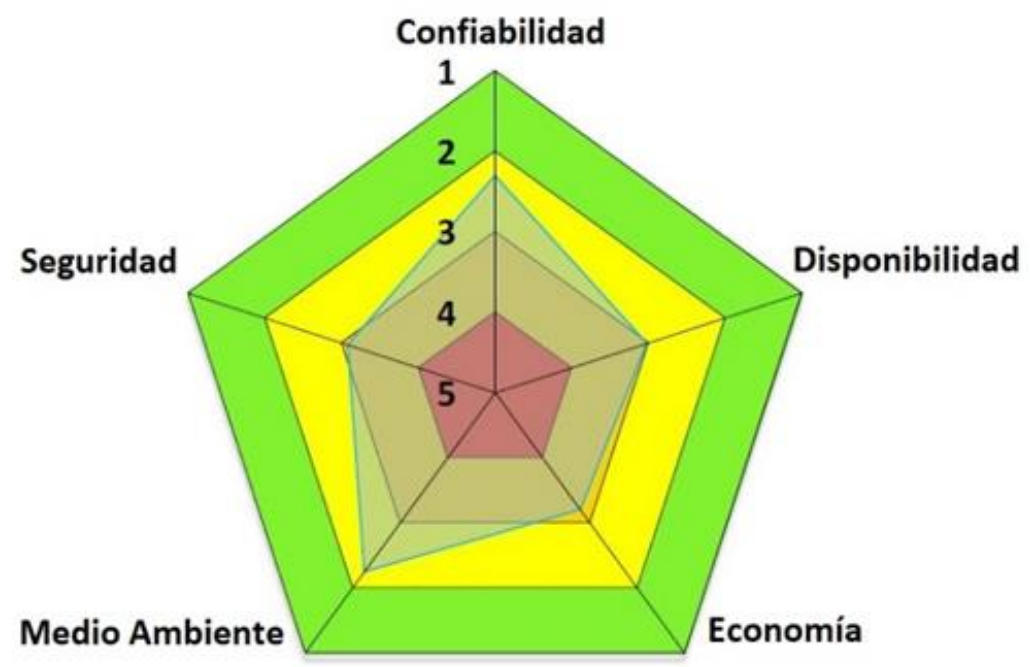

Figura 6: Diagrama de representación de resultados obtenidos mediante uso de KPI (COST Action TU1406, 2018)

El significado de la escala, por ejemplo, para el aspecto de Seguridad, es:

1 No hay peligro. Es muy poco probable que una persona pueda lesionarse debido al Desempeño actual del puente.

2 Es muy poco probable que una persona pueda lesionarse debido al Desempeño actual del puente.

3 Es poco probable que una persona pueda lesionarse debido al Desempeño actual del puente. La intervención se realizará antes de la próxima inspección.

$4 \quad$ Es probable que una persona pueda lesionarse debido al rendimiento actual del puente. La intervención se realizará poco después de la inspección.

$5 \quad$ Peligro inmediato. Es muy probable que una persona pueda lesionarse debido al Desempeño actual del puente. Se requiere acción inmediata.

En Chile, el Ministerio de Obras Públicas (MOP, 2018) aplica el "Manual de Carreteras", documento que es actualizado anualmente de acuerdo al Estado del Arte. En el Volumen 7 se incluye el capítulo sobre Puentes y Estructuras, describiendo cada una de las faenas, alcances y deterioros que se pueden encontrar dentro de los puentes. Adicionalmente, describe los procedimientos de trabajo para la reparación de cada uno de estos daños encontrados.

En este Manual se describe el procedimiento de Inspección de Puentes y Estructuras indicando los objetivos de dicho procedimiento. La idea principal de esta sección es caracterizar el procedimiento indicado cuyo objetivo es establecer el estado de serviciabilidad y estabilidad de la estructura, en 
función del nivel de deterioro existente, a través de la generación de un informe de inspección que debe ser analizado y revisado por un especialista de la Dirección de Vialidad para su validación. Estas inspecciones deben recoger información referente a:

- Detección de daños por acción ambiental, accidentes de vehículos, vandalismo y otros

- Actualización de información básica que posee la Dirección de Vialidad sobre las características de la obra (planos, estructuración, dimensiones, etc.)

- Evaluación del grado y nivel de deterioro que presenta la estructura para cuantificar los trabajos que deben realizarse

- Establecimiento de la prioridad de acciones a seguir para resguardo de la seguridad del puente y sus usuarios

- Programación de asignación de recursos para trabajos de mantenimiento y reparación necesarios Conforme a lo descrito en el Manual de Carreteras es posible apreciar que si bien existe un procedimiento estándar y caracterizado para evaluar los daños en los puentes, su carácter es de tipo cualitativo y no ofrece herramientas específicas que permitan obtener resultados objetivos y estandarizados de carácter cuantitativo para establecer el deterioro de las estructuras, lo cual resulta en una problemática debido a la variabilidad que pueden presentar los resultados obtenidos entre distintos profesionales que inspeccionen la estructura, sometiendo a la estructura a un ciclo de vulnerabilidad, por lo que se desprende la necesidad de incorporar otras herramientas al diagnóstico que permita tomar decisiones concretas, asertivas y oportunas

\subsection{Experiencias en Ensayos No-Destructivos END, Durabilidad y Vida Útil}

Dado el crecimiento en el uso del concreto en estructuras en el ámbito de instalaciones atómicas, y la ocurrencia de problemas debido a prácticas constructivas deficientes, la International Atomic Energy Agency (IAEA, 2002) preparó un Manual orientado al uso de las técnicas no-destructivas de Diagnóstico, debido a la poca experiencia existente en obras de concreto (el uso predominante de estas técnicas se daba en materiales metálicos).

Aparte de presentar los lineamientos básicos de una inspección, que incluye ensayos y Controles de Calidad, presenta en detalle los ensayos más habituales y los compara entre ellos, como:

$\begin{array}{ll}\circ & \text { Resistividad Superficial } \\ \circ & \text { Magnéticos - Espesor de Recubrimiento } \\ \circ & \text { Radares de Penetración GPR } \\ \circ & \text { Esclerómetros } \\ \circ & \text { Adhesión (Pull-Out) } \\ \circ & \text { Potencial Eléctrico de Media Celda } \\ \circ & \text { Velocidad de Corrosión } \\ \circ & \text { Profundidad de Carbonatación } \\ \circ & \text { Permeabilidad in-situ }\end{array}$

$\begin{array}{ll}\circ & \text { Penetración } \\ \circ & \text { Emisiones Acústicas } \\ \circ & \text { Ultrasonido } \\ \text { - Velocidad del Pulso } \\ \text { - Eco del Pulso } \\ \text { - Impacto-Eco-Frecuencia } \\ \circ \text { Termografía Infrarroja } \\ \circ \text { Métodos Radioactivos } \\ \circ \text { Métodos Nucleares }\end{array}$

Considerando la importancia de este tipo de ensayos en determinar las propiedades y desempeño de las estructuras de concreto en forma directa, algunos investigadores centraron sus esfuerzos en crear nuevos ensayos que permitiesen relacionar el desempeño de estructuras frente a agresiones del medio ambiente con mediciones realizadas directamente in-situ sobre la estructura (la mayoría de los ensayos de desempeño se ejecutan en condiciones de laboratorio). El trabajo fue exitoso (Torrent, R. Ebensperger, L., 1993) y la normativa suiza (SIA, 2003) procedió a incluir el ensayo de Permeabilidad al Aire como método válido a ser especificado en estructuras nuevas, con un control en la obra terminada a una edad entre 28 a 90 días.

Uno de los autores (Torrent, 2015) presentó un tiempo después la Metodología “Exp-Ref” que permite mediante mediciones no-destructivas realizadas en terreno, realizar estimaciones de Vida Útil/Residual de estructuras de hormigón. La metodología se basa en medir en la obra terminada las características del concreto de recubrimiento, y confrontarla a aquellos fenómenos ambientales agresivos, como el ingreso de carbonatación y/o cloruros. 
En los Análisis de Vida Útil se considera que el momento de inicio de la corrosión de la armadura de refuerzo corresponde al momento en que la capa protectora sobre el acero, que actúa depasivando la armadura frente a la corrosión $(\mathrm{pH}>12)$, es alcanzada por el frente de carbonatación o los cloruros, dando inicio al proceso electroquímico de la corrosión.

Por lo tanto, la durabilidad de la estructura estará dado por la capacidad resistente del concreto de recubrimiento para contrarrestar el ingreso de los agentes nocivos, y por la profundidad en que se ubique la armadura. Por esta razón, la metodología se basa en medir dos parámetros esenciales; la permeabilidad de esta capa de concreto y el espesor del recubrimiento. La Figura 4 muestra la relación que se da para una Vida Útil determinada entre la Permeabilidad al Aire y el Espesor del Recubrimiento, para una condición de ambiente con brisa salina (XS1-EN206). La zona sobre la curva cumple con el requisito de obtener una Vida Útil de Diseño a lo menos 50 años.

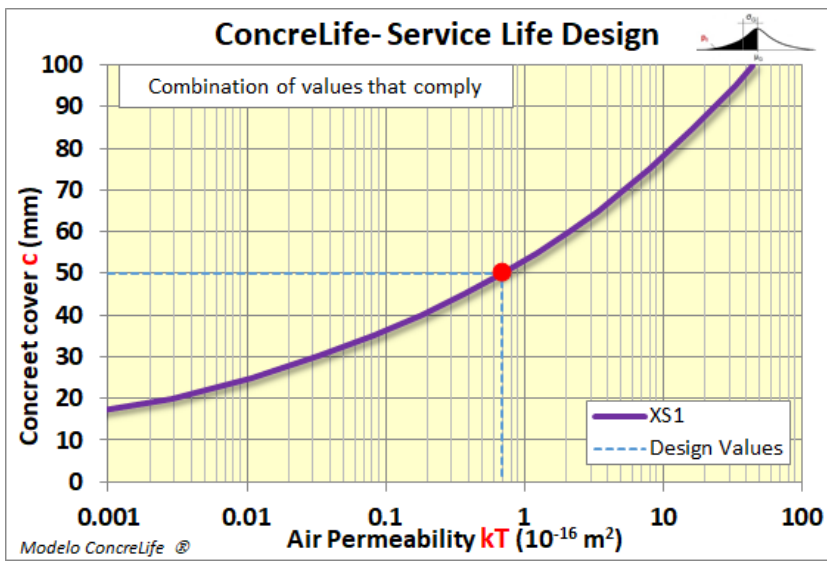

Figura 4: Relación entre Espesor y Permeabilidad del Recubrimiento para Ambiente Clase XS1

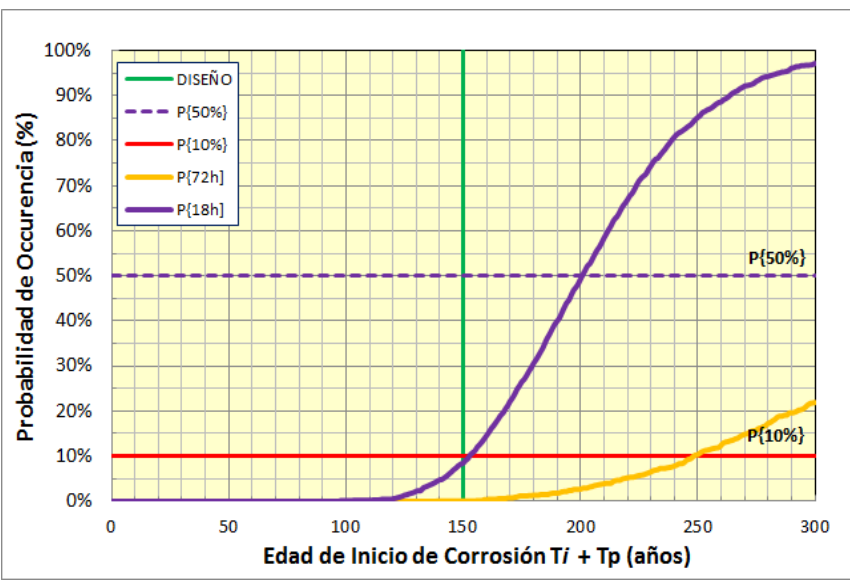

Figura 5: Probabilidad de inicio de la corrosión para un concreto determinado a partir de mediciones insitu

La posterior verificación en terreno de ambos parámetros sobre la estructura terminada, mediante la medición de suficientes pares de puntos para conocer la desviación estándar de cada medición, permite realizar estimaciones probabilísticas del inicio del proceso de corrosión del acero. La Metodología ha sido incorporada en el Modelo ConcreLife ${ }^{\circledR}$ (CTK, 2017) y permite realizar por un lado el DISEÑO de un concreto sujeto a distintos grados de severidad, ya sea por efecto de la acción de cloruros (Ambientes EN206 XS1, XS2 o XS3) o carbonatación (Ambientes EN206 XC1a, XC1b, XC2, XC3 o XC4), o por otro, el CONTROL en una obra terminada. La Figura 5 señala un ejemplo para este último caso: dovelas de concreto con una Vida Útil de Diseño de 150 años fueron curadas a vapor por un período de $18 \mathrm{~h}$ en vez del período establecido de $72 \mathrm{~h}$. El resultado entregó que la probabilidad de inicio de la corrosión para el curado de sólo 18h, a la edad de diseño de 150 años (curva morada), es de un $10 \%$, mientras que las otras dovelas lograrían esta misma probabilidad a los 250 años. En este ejemplo, ambos tipos de dovelas cumplieron con los requisitos establecidos y las de menor periodo de curado pudieron ser utilizadas en el proyecto.

Durante este año un grupo de investigadores (Di Pace et al, 2019) publican un nuevo enfoque aplicable al control del estado de estructuras de concreto durante su Ciclo de Vida. Para ello presentan las distintas etapas de un proyecto, y consideran las acciones que deben realizarse en cada una de ellas para asegurar un rango de serviciabilidad adecuado durante todo el período, además de un grado de resistencia frente a las acciones ambientales. Concluyen de la necesidad de participación de especialistas de distintas disciplinas en las siguientes etapas de un proyecto:

- Etapa de Anteproyecto: conocimiento acabado de las condiciones ambientales y datos de desempeño de los materiales disponibles en el sector. Idealmente se debiera contar con un historial de desempeño de éstos. 
- Etapa de Proyecto: se aplican modelos de predicción de deterioro que aseguren la obtención de la vida útil requerida. La Metodología de la fib son el "Model Code for Service Life Design" para el Diseño por Vida Útil es la comúnmente usada a nivel mundial. Se definen los materiales a utilizar, como tipo de cemento (prevención de deterioros internos), la mezcla de concreto óptima (desempeño probados para obtener elevados niveles de resistencia a los agentes agresivos), se especifican las técnicas y prácticas constructivas que aseguren la obtención de un recubrimiento impermeable y de espesor adecuado, y por último, se diseña el sistema de control de calidad, disponible para detectar situaciones anómalas, que puedan ser subsanadas a la brevedad, sin que interfieran en la obtención de la vida útil proyectada.

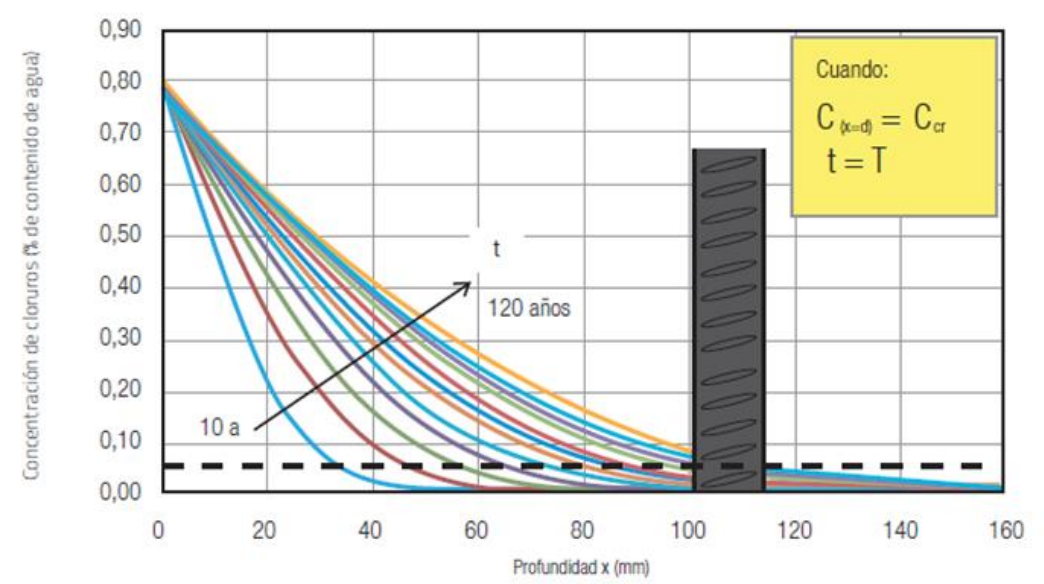

Figura 6: Análisis de Vida Útil durante la Etapa de Proyecto para distintas edades, con el objetivo de determinar edad en que el perfil de cloruros alcanza la profundidad del acero de refuerzo

- Etapa de Construcción: se verifica que los materiales y mezclas de concreto cumplan los requisitos mediante un Control de Calidad rutinario. Se ejecutan ensayos idealmente tipo END no-destructivos sobre la estructura terminada, a modo de corroborar el cumplimiento del desempeño definido en la Etapa de Diseño, como por ejemplo el indicado en el punto anterior. El uso de modelos, como el presentado en párrafos anteriores, permiten validar el estado de la estructura recién construida.

- Etapa de Entrega de la Obra: se elabora un "Certificado de Nacimiento" que muestra la situación de calidad de la estructura en el momento de la entrega. Contiene todos los resultados de control de calidad, tanto de laboratorio como de campo, indicando las zonas más vulnerables, bien sea como consecuencia de su grado de exposición o de sus características y propiedades. Se entrega, además, un "Manual de Mantenimiento" que detalla las acciones a llevar a cabo durante la vida útil de la estructura a fin de prevenir con la máxima antelación eventuales deterioros y remediarlos oportunamente.

- Etapa de Operación: se ejecutan Planes de Mantenimiento y de Diagnóstico establecidos para el control en el tiempo de la estructura.

- Etapa de Devolución de Concesiones: es necesario en muchos casos conocer la Vida Residual de la estructura.

\section{DESARROLLO METODOLOGÍA Y MODELO}

La aplicación de procedimientos y ensayos in-situ en las estructuras, permite obtener datos objetivos del estado de los diversos elementos de una estructura, que permitirían conocer en forma muy rápida su nivel de integridad y proponer acciones correctivas más precisas sobre las medidas a adoptar. En particular, el uso de equipos de ensayos no-destructivos que permitan conocer la resistencia del hormigón, el potencial de corrosión, la ubicación de las armaduras, el recubrimiento, entre otros, ayudan 
a completar la información requerida sobre el estado de la estructura, en términos que facilita la labor del profesional a cargo de la inspección, debido a que reduce los niveles de incertidumbre que genera la evaluación tan solo visual del daño, sin ensayos que permitan corroborar con niveles de exactitud mayor el estado real de la estructura.

Pensar en una aplicación simultánea de estos ensayos in-situ, entrega sin dudas un volumen de información mayor para la realización de un mejor Diagnóstico. Esta es la experiencia que los autores han obtenido en los últimos años (Ebensperger, L., Donoso, J.P., 2018) en diferentes trabajos a lo largo del país, y que se resumen en la Figura 7:
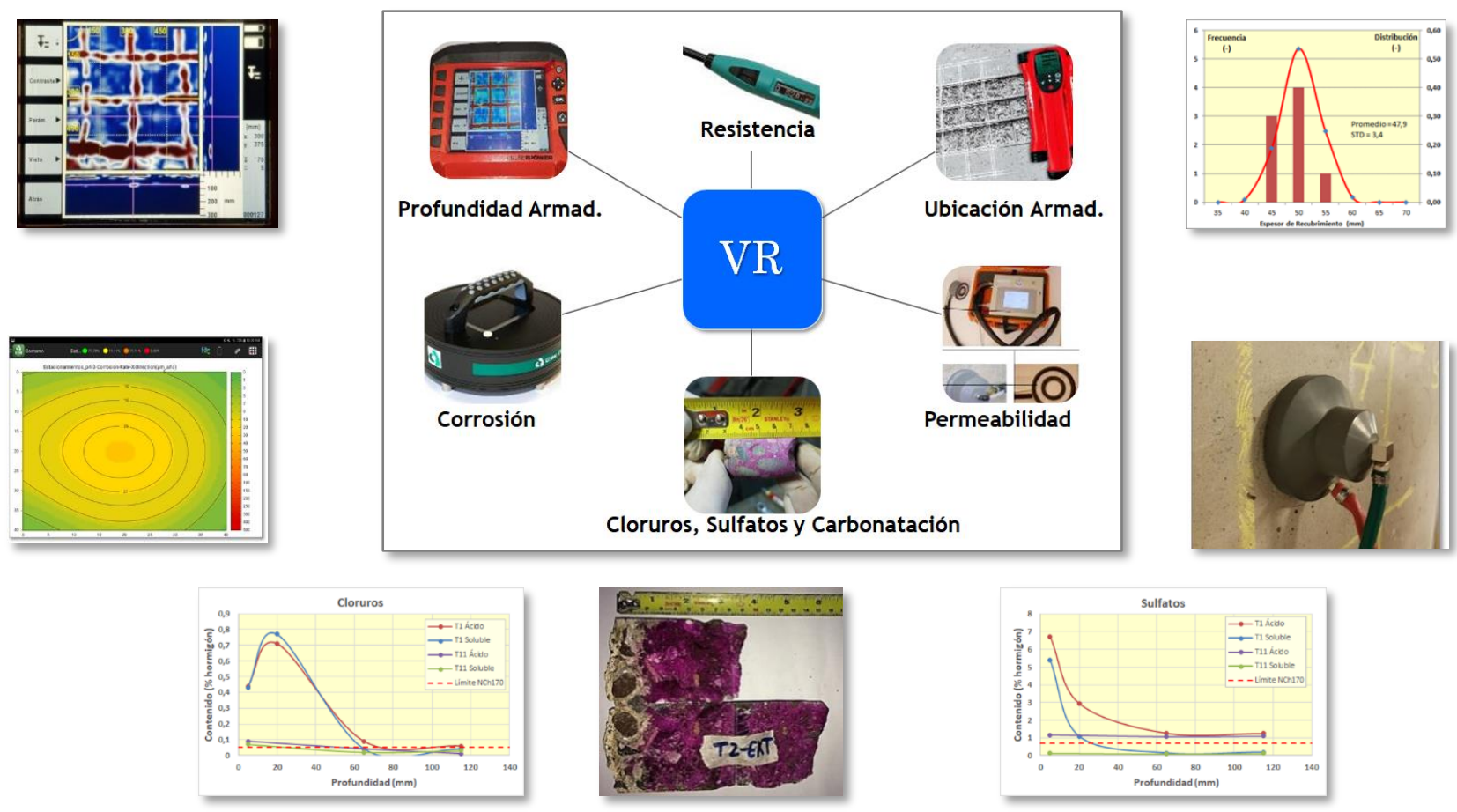

Figura 7: Utilización de diferentes técnicas para estimar la Vida Residual de estructuras de concreto

A partir de algunos trabajos desarrollados en Europa (COST Action TU1406, 2018), se formuló el concepto de la Metodología a desarrollarse, la cual debía consistir en la formulación de un Nivel de Deterioro Global de una Estructura, a determinarse mediante el Índice de Deterioro a través de distintos ensayos no-destructivos y la incorporación del Índice de Severidad de la Exposición Ambiental que afecta a la estructura.

Se propone en una primera instancia la inclusión de 14 parámetros de Deterioro y una adaptación de los Grados de Severidad de la normativa EN206. A partir de la ponderación de cada Índice y el posterior cálculo del promedio de ambos, se determina el Nivel de Deterioro Global de la Estructura NDG. Este último valor podrá ser comparado en el tiempo, a medida que nuevos Diagnósticos sean realizados, y el Nivel que alcance en cada caso determinará las acciones a ejecutar en el elemento estructural evaluado.

Para cada ensayo y Exposición Ambiental se definen hasta 4 niveles de deterioro y severidad, respectivamente, de acuerdo a la práctica, la cual podría variar de un país a otro. El objetivo de la 3era. parte de este trabajo, próxima a realizarse, es justamente validar los niveles propuestos que sean elegidos en cada caso. La Figura 8 muestra una primera aproximación de la Metodología desarrollada, a la cual se le ha denominado Modelo CTK-ConDiag® (CTK, 2018). 
Índice de Deterioro - ID

\begin{tabular}{|c|c|c|c|c|c|c|}
\hline \multirow{2}{*}{ Ensayo } & \multirow{2}{*}{ Deterioro } & \multirow{2}{*}{ Unidad } & \multicolumn{4}{|c|}{ Índice de Deterioro } \\
\hline & & & 1 & 2 & 3 & 4 \\
\hline 1 & Contenido de Aire Concreto & $\%$ & 36 & $6-4$ & $4-2$ & $<2$ \\
\hline 2 & Grietas, ancho & $\mathrm{mm}$ & $<0.05$ & $0.05-0.30$ & $0.30-1.0$ & $1.0-3.0$ \\
\hline 3 & Humedad del Concreto & $\%$ & $<2$ & $2-4$ & 4.6 & $>6$ \\
\hline 4 & Profundidad de Carbonatación & $\mathrm{mm}$ & 0 & $<c$ & $=c$ & $>c$ \\
\hline 5 & Frente de Cloruros & $\mathrm{mm}$ & 0 & $<c$ & $=c$ & $>c$ \\
\hline 6 & Cloruros a nivel de refuerzo & \% peso hor. & $<0.025$ & $0.025-0.030$ & $0.025-0.030$ & $>0.040$ \\
\hline 7 & Resistividad & $\mathrm{Wm}$ & $>200$ & $200-100$ & $100-50$ & $<50$ \\
\hline 8 & Potencial de Celda Corrosión (Cu) & $\mathrm{mV}$ & $>-200$ & $.200 \quad-350$ & $-350-450$ & $<-450$ \\
\hline 9 & Velocidad de Corrosión & $\mu \mathrm{A} / \mathrm{cm}^{2}$ & $<1$ & $1-3$ & $3-10$ & $>10$ \\
\hline 10 & Diámetro Aceros & $\mathrm{mm}$ & $>20$ & $20-16$ & $16-12$ & $<12$ \\
\hline 11 & Pérdida de masa del acero & $\%$ & $<2$ & $2-5$ & $5-10$ & $>10$ \\
\hline 12 & Resistencia del Concreto & $\mathrm{MPa}$ & $>35$ & $>30$ & $>25$ & $<20$ \\
\hline 13 & Espesor del Recubrimiento & $\mathrm{mm}$ & $>65$ & $60-40$ & $40-20$ & $<20$ \\
\hline 14 & Permeabilidad al Aire del Recub. & $10^{-16} \mathrm{~m}^{2}$ & $<0.010$ & $0.010-1.0$ & $1-10$ & $>10$ \\
\hline
\end{tabular}

Índice de Exposición Ambiental - IEA

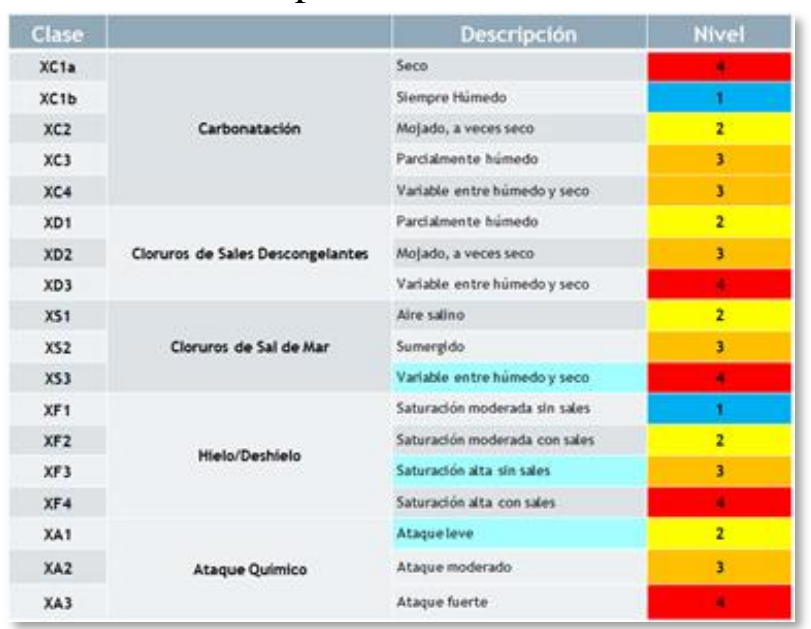

Las celdas marcadas con un color celeste corresponden al caso de un ejemplo: estructura ubicada en clima frío con alta humedad sin el uso de sales, en ambiente marino severo y con ataque químico leve. De las mediciones realizadas, se determinó valores de 4 en el Potencial de Corrosión, el espesor de Recubrimiento y la Permeabilidad al Aire. Con un ID=2.62 y un IEA=3.00 el NDG=2.82, alcanzando un Nivel Tipo V con un Deterioro muy Alto.

\begin{tabular}{|c|c|c|}
\hline Nivel de Deterioro & Descripción & NDG \\
\hline I & Sin Deterioro & $<0,65$ \\
\hline II & Deterioro Menor & $0,65-1,20$ \\
\hline III & Deterioro Medio & $1,20-1,90$ \\
\hline IV & Deterioro Alto & $1,90-2,55$ \\
\hline V & Deterioro muy Alto & $2,55-3,50$ \\
\hline VI & Deterioro Crítico & $>3,50$ \\
\hline
\end{tabular}

Figura 8: Presentación de Metodología de Diagnóstico Integral de Estructuras de Concreto Reforzado

\section{CONCLUSIONES}

Tras un acucioso estudio bibliográfico sobre los alcances de la ejecución de un Diagnóstico a una estructura de concreto reforzado, unido a la experiencia de los autores en la ejecución de trabajos de esta índole, fue posible determinar que, para obtener una evaluación de calidad del estado actual de una estructura, se requiere incorporar en forma complementaria a la inspección visual la ejecución de ensayos no-destructivos. Se propone una Metodología que integra la condición de exposición ambiental, y que través del manejo de índices, permite obtener un Nivel de Deterioro Global de una estructura. Esta Metodología debe ser ratificada con pruebas en terreno. Se espera que los resultados de la aplicación de esta metodología aporten a las herramientas actuales de diagnóstico inicial, mejorando la toma de decisión por parte de los profesionales involucrados.

\section{AGRADECIMIENTOS}

La ejecución de parte de este trabajo ha sido financiada con aportes de la Comisión Nacional de Investigación Científica y Tecnológica CONICYT-Chile, corporación orientada al desarrollo científico 
y tecnológico. Las actividades de terreno a su vez cuentan con la colaboración del Departamento de Puentes de la Dirección de Vialidad del Ministerio de Obras Públicas de Chile.

\section{BIBLIOGRAFÍA}

AAHSTO (2011): “MBE- Manual for Bridge Evaluation”, 2nd. Edition AASHTO (2017): "LRFD Bridge Design Specifications", 8th Edition ACI 364.1 R-07 (2007): "Guide for Evaluation of Concrete Structures Prior to Rehabilitation" ACI 201.1 R-08 (2008): "Guide for Conducting a Visual Inspection of Concrete in Service" ACI 562-13 (2013): “Code Requirements for Evaluation, Repair, and Rehabilitation of Concrete Buildings and Commentary"

CTK Construtechnik (2017), "Modelo ConcreLife®-Concrete Service Life Design\&Control" CTK Construtechnik (2018), "Modelo ConDiag® - Concrete Diagnosing"

Di Pace, G., Ebensperger, L., Torrent, R., Bueno, V. (2019), "From Cradle to Maturity - A holistic service-life approach for concrete bridges", Concrete International, April 2019.

Ebensperger, L., Donoso, J.P. (2018), "Diagnóstico Estructural estructuras existentes", Informes Privados de empresas.

European Cooperation in Science \& Technology COST Action TU1406 (2018): "Quality Specifications for Roadway Bridges, standardization at a European Level"

European Standard EN206 (2013): "Concrete - Specification, performance, production and Conformity- Exposure classes related to environmental actions"

Imamoto, K. (2016): “JCI Guidelines for Assessment of Existing Concrete Structures", Seminario Internacional de Estructuras de Hormigón", F. de Ingeniería - Univ. de Buenos Aires.

ISO 16311 (2014): "Maintenance and repair of concrete structures"

International Atomic Energy Agency (2002): "Guidebook on NDT of Concrete Structures".

Ministerio de Obras Públicas (2018): "Manual de Carreteras", Volumen 7, Edición 2018

Torrent, R., Exp-Ref (2015): "A Simple, Realistic and Robust Method to Assess Service Life of Reinforced Concrete Structures", Concrete 2015, September, Melbourne, Australia.

SIA 261/1 - Anhang E (2003, 2013): "Luftpermeabilität am Bauwerk".

Torrent, R., Ebensperger, L. (1993), "Study on Methods to Measure and Evaluate the Characteristics of the Cover Concrete on Site," Report 506, Swiss Federal Highways Office, Bern, Switzerland. 